\title{
Malabsorption and artificial nutrition in patients with gut GvHD post allogenic stem cell transplantation: Home parenteral nutrition affects survival significantly
}

\author{
Konstantinos C. Fragkos ${ }^{1}$, Hanson Kwok ${ }^{1}$, Aaron Bhakta ${ }^{1}$, Niamh Keane ${ }^{1}$, Ronjon Chakraverty ${ }^{2}$, Kirsty \\ Thompson ${ }^{2}$, Farooq Rahman ${ }^{1}$, Simona Di Caro ${ }^{1}$, Shameer J Mehta ${ }^{1}$ \\ ${ }^{1}$ Intestinal Failure Unit, GI Services, UCLH, UK \\ ${ }^{2}$ Department of Haem-Oncology, UCLH, UK
}

Rationale: Gut graft versus host disease (GvHD) in patients post stem cell transplantation is associated with malabsorption and malnutrition. The purpose of this study was to examine the causes of malnutrition and assess modes of delivery of artificial nutritional support.

Methods: This was a retrospective study of patients who had undergone allogenic stem cell transplantation from January 2009 to December 2017 at UCLH and were referred to the hospital nutrition support team with gut GvHD.

Results: Eighteen patients were included ( 6 females, mean age $44.6 \pm 17.6$ years). Median time to gut GvHD post-transplant was 190 days [IQR 80-261]. Clinical grade 3 and 4 gut GvHD was present in $72.2 \%$ and 27.8\% of patients respectively. Diarrhoea was present in all patients, with bloating (5.6\%) and rectal bleeding (11.1\%) being less common. Fifty percent of patients had imaging features of colitis, typhlitis, ileitis, pneumatosis coli or stricturing disease. Histology indicated patchy inflammation in all patients. Gut GvHD was treated with methylprednisolone in all patients and infliximab in $38.9 \%$ of patients, while one patient received both ruxolitinib and vedolizumab. All patients had tried oral nutritional supplements, $44.4 \%(n=8)$ enteral nutrition (EN), 55.6\% $(n=10)$ parenteral nutrition (PN) and $66.7 \%(n=12)$ EN or PN; $27.8 \%$ went home on PN. Main causes of malabsorption were small intestinal bacterial overgrowth (hydrogen breath test positive in $6 / 7)$, bile acid diarrhoea $(11.1 \%, 2 / 18)$, and pancreatic exocrine insufficiency (elastase $<200$ in 2/8). Mean BMI was $18.8 \mathrm{~kg} / \mathrm{m} 2$ pre and post EN/PN. For patients on home PN, the average daily script was volume $2039 \mathrm{~mL}$, calories $1689 \mathrm{kcal}$ (lipid $737 \mathrm{kcal}$ ), nitrogen $10.3 \mathrm{~g}$, sodium $154 \mathrm{mmol}$, potassium $62 \mathrm{mmol}$, calcium $7.4 \mathrm{mmol}$, magnesium $8.8 \mathrm{mmol}$, and phosphate $21 \mathrm{mmol}$. Median survival was 187 days [IQR 1021084]. Survival was not different between those who had tried EN or PN and those who had not (log rank $p>0.05$ in both cases) but survival was better in patients who had received home PN against those who had not ( $\log$ rank $p=0.017)$.

Conclusion: This is the first study to examine cases of gut GvHD and associated nutritional sequelae. Clinicians should be alerted to these causes of malabsorption and refer to nutrition support teams.

Disclosure of Interest: None declared. 


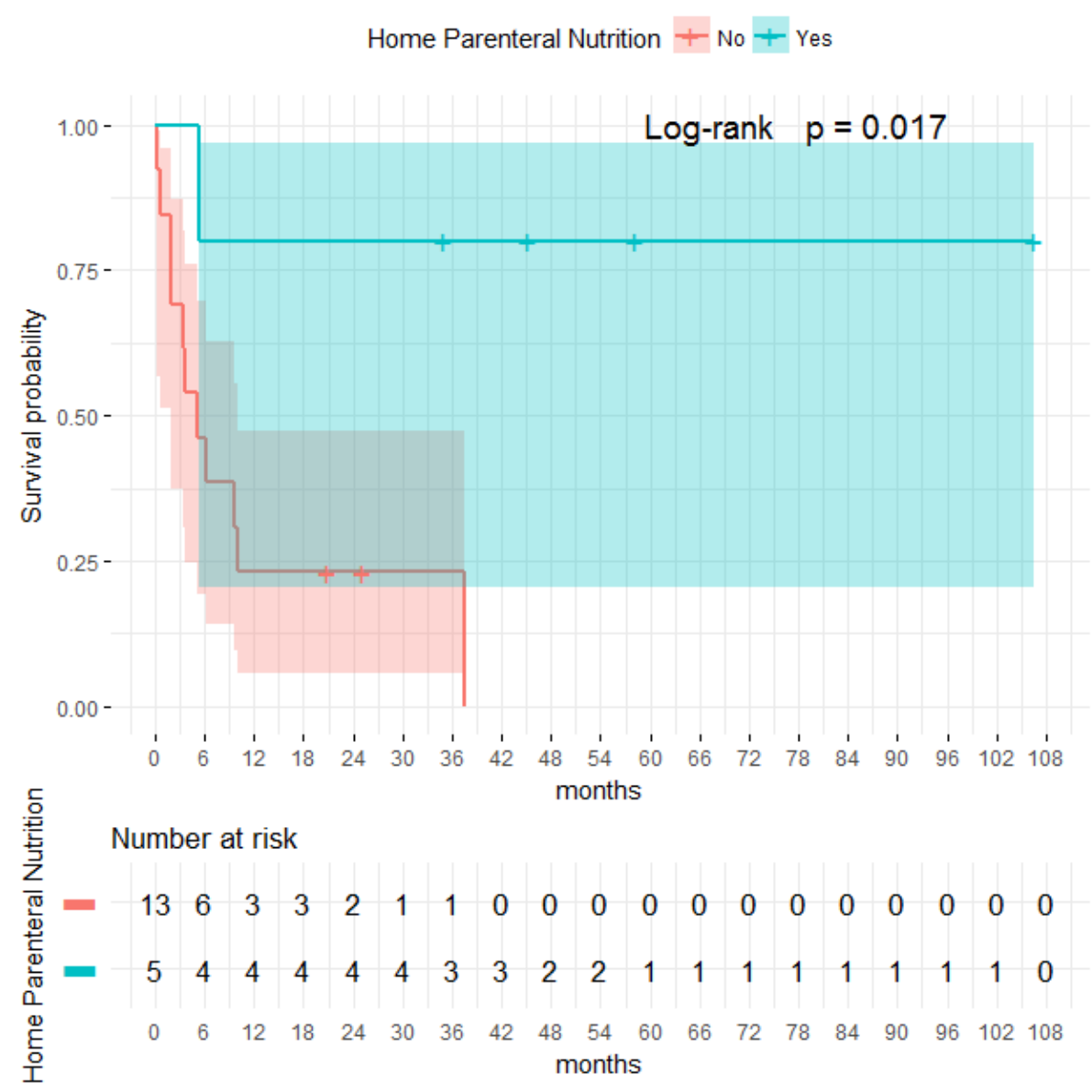

